\title{
Article
}

\section{Evaluation of 13 rapidly-mutating Y-STRs in endogamous Punjabi and Sindhi ethnic groups from Pakistan}

\author{
Hadi, Ss
}

Available at http://clok.uclan.ac.uk/25409/

Hadi, Ss ORCID: 0000-0002-2994-3083 (2019) Evaluation of 13 rapidlymutating Y-STRs in endogamous Punjabi and Sindhi ethnic groups from Pakistan. International Journal of Legal Medicine, 133 (3). pp. 799-802. ISSN $0937-9827$

It is advisable to refer to the publisher's version if you intend to cite from the work. http://dx.doi.org/10.1007//500414-018-01997-9

For more information about UCLan's research in this area go to http://www.uclan.ac.uk/researchgroups/ and search for < name of research Group>.

For information about Research generally at UCLan please go to http://www.uclan.ac.uk/research/

All outputs in CLoK are protected by Intellectual Property Rights law, including Copyright law. Copyright, IPR and Moral Rights for the works on this site are retained by the individual authors and/or other copyright owners. Terms and conditions for use of this material are defined in the policies page.

\section{CLoK}

Central Lancashire online Knowledge www.clok.uclan.ac.uk

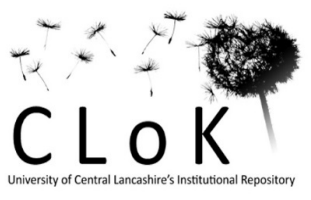




\title{
Evaluation of 13 rapidly-mutating Y-STRs in endogamous Punjabi and Sindhi ethnic groups from Pakistan
}

\begin{abstract}
:
Y-chromosomal short tandem repeats (Y-STRs) are commonly used to conclude population histories, discover ancestral relationships, and identify males for criminal justice purposes. YSTRs being largely in forensic use have low haplotype diversity in some populations and fail to discriminate between male relatives. Rapidly mutating Y-STRs (RM Y-STRs) were breakthrough of this decade and have been paid much attention. A set of 13 rapidly-mutating (RM) Y-STRs (DYF387S1, DYF399S1, DYF403S1a/b1/b2, DYF404S1，DYS449，DYS518，DYS526I/II, DYS547, DYS570, DYS576, DYS612, DYS626, and DYS627) typically reveals higher haplotype diversities than the commercially available Y-STR sets and allows differentiating male relatives for which commercial Y-STR sets are usually not informative. Here, we amplified the 13 RM YSTRs in 168 (37 Sindhi and 131 Punjabi) individuals from Pakistan, which is characterized by high rates of endogamy. The haplotype diversity and discrimination capacity were 1 . Allelic frequencies ranged from 0.0060 to 0.5060 , while gene diversity ranged from 0.6759 (DYS526a) to 0.9937 (DYF399S1). A total 319 unique alleles were observed. Results of our study showed that RM Y-STRs provided substantially stronger discriminatory power in Pakistani populations.
\end{abstract}

Key Words: RM Y-STRs, Paternal Lineage, Endogamous, Punjabi, Sindhi, Pakistan

Pakistan has one of the highest reported rates of consanguineous marriages in the world. More 
than half of all marriages $(61 \%)$ are between first and second cousins. First-cousin marriages are more common on the father's side (32\%) but also occur between first cousins on the mother's side $(21 \%)$. Eight percent of marriages are between second cousins, 7 percent are between other relatives, and one-third are between non-relatives. Differences in marriage patterns are observable by urban-rural areas. First-cousin marriages are most common in rural areas (57 percent) and are less common in major cities where about 40 percent of marriages are between first cousins (http://www.pbscensus.gov.pk). These mentioned trends make Pakistani population endogamous population and to differentiate between related and unrelated male individuals is a challenge. Y chromosome STRs are widely used in human population[1] sexual offences, paternity testing and missing person investigations[2] because it shows a male inheritance and substantial structuring in human populations[3]. For these applications the currently available YSTR panels (Y-filer \& Y23) have proven to be effective tools with numerous case reports[4-6]. No doubt currently used Y STR markers holds appropriate potential for the reconstruction of human population history, $[7,8]$ including estimation of demographic parameters [9],as well as for genealogical relationships[10] and male lineage determination in forensic applications[8-10] There are some limitations to their use in forensic investigations. The capacity to discriminate between individuals of same paternal linage is considerably less than that of the autosomal STR sets used in forensics. While this is primarily due to the uniparental inheritance and lack of recombination of male-specific Y-chromosome markers in general, the particular YSTRs currently in forensic use also contribute to the incomplete haplotype resolution. A number of studies have demonstrated that Y-STR haplotype diversity, as measured with current Y-STR sets, can be increased and male lineage differentiation can be improved by adding carefully ascertained additional Y-STRs [14-16] 
A set of 13 Y-STRs with high mutation rates has been classified when compared with the Y-STRs commonly used (Y-filer \& Y23) in genetic forensics. These markers (DYF399S1, DYF387S1,DYS570, DYS576, DYS518, DYS526a+b, DYS626, DYS627, DYF403-S1a+b1+b2, DYF404S1, DYS449, DYS547 and DYS612), denominated rapidly mutating Y-STRs (RM YSTRs), promise to be an important tool in the differentiation of two male individuals closely related. Blood samples were randomly collected from unrelated 131 Punjabi and 37 Sindhi individuals with informed consent after the approval of ethical review board of China Medical University Shenyang China. DNA was isolated from blood using the ReliaPrep ${ }^{\mathrm{TM}}$ Blood gDNA Miniprep System (Promega, Madison, USA) according to the manufacturer's instructions. The 13 RM Y-STR were amplified in 2 multiplex PCR reactions: RM1 (amplifying DYF399S1, DYS518, DYS547, DYS526.2, DYS626, DYS526, DYF404S1, DYS627), and RM2 (amplifying DYS612, DYF387S1, DYS547, DYS570, DYS449, DYS576, DYF403S1a, DYF403S1b, DYF403S1b2) as described previously [17]. Arlequin V 3.5.2 package software [13] used to calculate the gene diversity (GD), haplotype frequencies and allelic frequencies. Match probabilities (MP) were calculated as the sum of squared haplotype frequencies. The discrimination capacities (DC) were calculated by dividing the number of different haplotypes by the total number of samples.

We successfully obtained genotypes of 168 individuals from Pakistan are listed in supplementary Table 1. Allelic frequencies and gene diversity values for all 13 RM Y-STRs are listed in Supplementary Table 2. A total of 223 alleles were observed for four multi-copy RM Y STRs while in total 319 different alleles were observed for 13 RM Y STRs. DYF399S1 was most diverse marker with 114 alleles and DYF404S1 was the lest diverse with 22 alleles in 4 muticopy markers among 13 RM Y STRs. Allelic frequencies ranged from 0.0060 to 0.5060 while gene diversity (GD) ranged from 0.6759 (DYS526a) to 0.9937 (DYF399S1) and over all GD 
value was 0.8347 . For forensic statistics analysis we have evaluated our data at eight different levels (Table 1). For the whole set of 13 RM Y-STRs we have observed 168 haplotypes, every haplotype was unique with haplotype diversity 1while random matching probability (RMP) 0.0060 and discrimination capacity was also 1 . When we excluded all multicopy markers from analysis HD value decreased to 0.9992 with 152 haplotypes among them 152 were unique with DC value was 0.9464 and RMP value was 0.0067. Thermo Fisher Scientific, MA, USA company introduced a new Yfiler plus kit which contains 27 Y STRs loci in which 6 are RM Y STRs. So we also evaluated our data on these 6 RM Y STRs and we have observed 147 haplotypes with GD value 0.9982 , DC value 0.8750 and RMP 0.0077. Among 147 haplotypes 130 were unique and 13 haplotypes were shared in 2 individuals and 4 haplotypes were shared in 3 individuals. In conclusion the analysis of the data showed that all the 168 samples had unique haplotypes. It was evidently revealed from a value of haplotype diversity equal to 1 . These results are in agreement with previous studies, which indicate that the RM Y-STR set could considerably increase the discrimination power compared with the markers commonly used in YSTR commercial kits[18]. However, further studies should be conducted in the populations of this region, mainly in comparison with commercial kits to prove these claims. Nevertheless, the main purpose to contribute to increase the global database for 13 RM Y-STR set was fulfilled.

References: 
1. Underhill PA, Kivisild T (2007) Use of y chromosome and mitochondrial DNA population structure in tracing human migrations. Annu Rev Genet 41:539-564.

https://doi.org/10.1146/annurev.genet.41.110306.130407

2. Kayser M (2007) Uni-parental markers in human identity testing including forensic DNA analysis. BioTechniques Supplement to Vol. 43, No. 6, December 2007:Sxv-Sxxi

3. Karafet TM, Mendez FL, Meilerman MB, et al (2008) New binary polymorphisms reshape and increase resolution of the human Y chromosomal haplogroup tree. Genome Res 18:830-838. https://doi.org/10.1101/gr.7172008

4. Adnan A, Rakha A, Noor A, et al (2017) Population data of 17 Y-STRs (Yfiler) from Punjabis and Kashmiris of Pakistan. Int J Legal Med. https://doi.org/10.1007/s00414-0171611-9

5. Adnan A, Rakha A, Kasim K, et al (2018) Genetic characterization of Y-chromosomal STRs in Hazara ethnic group of Pakistan and confirmation of DYS448 null allele. Int J Legal Med. https://doi.org/10.1007/s00414-018-1962-X

6. Atif Adnan, Kasim K, Rakha A, et al (2018) Population data of 23 Y STRs from Manchu population of Liaoning Province, Northeast China. Int J Legal Med. https://doi.org/10.1007/s00414-018-1957-7

7. Kayser M, Brauer S, Weiss G, et al (2000) Melanesian origin of Polynesian Y chromosomes. Curr Biol CB 10:1237-1246

8. Underhill PA, Shen P, Lin AA, et al (2000) Y chromosome sequence variation and the history of human populations. Nat Genet 26:358-361. https://doi.org/10.1038/81685

9. Shi W, Ayub Q, Vermeulen M, et al (2010) A worldwide survey of human male demographic history based on Y-SNP and Y-STR data from the HGDP-CEPH populations. Mol Biol Evol 27:385-393. https://doi.org/10.1093/molbev/msp243

10. Kayser M, Vermeulen M, Knoblauch H, et al (2007) Relating two deep-rooted pedigrees from Central Germany by high-resolution Y-STR haplotyping. Forensic Sci Int Genet 1:125-128. https://doi.org/10.1016/j.fsigen.2007.02.004

11. Kayser M, Caglià A, Corach D, et al (1997) Evaluation of Y-chromosomal STRs: a multicenter study. Int J Legal Med 110:125-133, 141-149

12. Dettlaff-Kakol A, Pawlowski R (2002) First Polish DNA "manhunt"--an application of Ychromosome STRs. Int J Legal Med 116:289-291. https://doi.org/10.1007/s00414-0020320-0

13. Roewer L (2009) Y chromosome STR typing in crime casework. Forensic Sci Med Pathol 5:77-84. https://doi.org/10.1007/s12024-009-9089-5 
14. Hanson EK, Ballantyne J (2004) A highly discriminating 21 locus Y-STR "megaplex" system designed to augment the minimal haplotype loci for forensic casework. J Forensic Sci 49:40-51

15. Hanson EK, Ballantyne J (2007) An ultra-high discrimination $Y$ chromosome short tandem repeat multiplex DNA typing system. PloS One 2:e688. https://doi.org/10.1371/journal.pone.0000688

16. Vermeulen M, Wollstein A, van der Gaag K, et al (2009) Improving global and regional resolution of male lineage differentiation by simple single-copy Y-chromosomal short tandem repeat polymorphisms. Forensic Sci Int Genet 3:205-213.

https://doi.org/10.1016/j.fsigen.2009.01.009

17. Lee EY, Lee HY, Kwon SY, et al (2016) A multiplex PCR system for 13 RM Y-STRs with separate amplification of two different repeat motif structures in DYF403S1a. Forensic Sci Int Genet 26:85-90. https://doi.org/10.1016/j.fsigen.2016.10.019

18. Adnan A, Ralf A, Rakha A, et al (2016) Improving empirical evidence on differentiating closely related men with RM Y-STRs: A comprehensive pedigree study from Pakistan. Forensic Sci Int Genet 25:45-51. https://doi.org/10.1016/j.fsigen.2016.07.005 